\title{
INTERVENTION EFFECT OF TIME MANAGEMENT TRAINING ON NURSES' MENTAL HEALTH DURING THE COVID-19 EPIDEMIC
}

\author{
Liuyu Sun \\ School of Economics \& Management, Tongji University, Shangha, China \\ Department of Business and Management, Shanghai Industrial and Commercial Polytechnic, Shanghai, China
}

received: 21.5.2021;

revised: 14.8.2021;

accepted: 17.9.2021

\section{SUMMARY}

Background: The sudden outbreak of COVID-19 has put nurses into a severe test, both physiologically and psychologically. While being required to provide patients with high-quality medical services, nurses also bear the responsibilities and pressures from work, face trauma, disease and even death events, and are thereby more inclined to negative psychological feelings, decline in mental health, and reduction in the quality of their clinical nursing services. Under the background of the COVID-19 epidemic, it is urgent to carry out related intervention in nurses' psychological crisis.

Subjects and methods: The mental health of 400 nurses from three tertiary hospitals in Shanghai, China was assessed from September to December 2020. Then, time management training was conducted for 66 nurses who were voluntarily enrolled in the study. They were divided into the intervention group (35 participants) and the control group (31 participants).

Results: After the 16-week intervention, (1) there is a significant decrease in the total SCL-90 score of the intervention group and significant decreases in the scores in the 9 dimensions of the scale, suggesting significant improvement in the mental health level; (2) there is a significant increase in the score of the intervention group in subjective well-being, while there is no significant increase in the control group; (3) There is a significant decrease in the score of the intervention group in work stress reaction, but there is no significant decrease in the control group, and there is a significant increase in the physiological reaction of the control group in the measurement after 8 weeks.

Conclusions: It is critical to pay attention to and solve the low mental health level of nurses during the COVID-19 epidemic; Time management training can effectively improve the mental health level of nurses, and it is an effective intervention model to promote nurses' mental health and relieve their work stress.

Key words: mental health - time management training - Balint group intervention - medical nurses

$* * * * *$

\section{INTRODUCTION}

As of May 2021, the accumulated number of confirmed cases infected with COVID-19 worldwide has exceeded 150 million, approaching 160 million, while the accumulated number of deaths has surpassed 3 million. Although the number of infected cases and the number of deaths have been reducing significantly as a result of the rapid, effective implementation of epidemic prevention measures, it is still necessary to reinforce the epidemic prevention work in consideration of the existing confirmed cases. Evidently, the COVID-19 epidemic has brought serious impact to the general public. It was revealed in a study that many ordinary people reported to have post-traumatic stress disorder (PTSD), and their PTSD symptoms were at the highest level in the peak of diagnosed infection with COVID-19 and then decreased gradually (Wang et al. 2020). Noticeably, COVID-19 has not only affected the mental health of ordinary people, but also posed unprecedented challenges to the mental health of nurses, who are important practitioners in the fight against the COVID19 epidemic. In terms of nursing work, it has been shown in previous studies that nurses have the highest perceived occupational stress compared with other workers (Labrague et al. 2018). After the outbreak of the epidemic, most nurses have significant increase in the average working hours, leading to the serious deterioration of their mental stress level and perceived work stress (Davidson et al. 2020, Mosolova et al. 2020, Salopek-Ziha et al. 2020). It was found in a study that the prevalence of depression in nurses engaged in clinical work reaches $22.8 \%$, with the prevalence of anxiety disorder being $23.2 \%$ and the prevalence of sleep disorder being 38.9\% (Hofmeyer et al. 2021). In particular, female nurses tend to have more severe emotional disorders (Pappa et al. 2020). In another review study, the group of nurses was also verified to exhibit higher levels than other groups in terms of work stress, depression, anxiety and sleep disorders (Al Maqbali et al. 2020). In an interview-dominated investigation on nurses' psychological changes during the COVID-19 epidemic, it was also pointed out that some nurses are afraid of being infected in the early psychological stage, and most nurses suffer from anxiety, depression, somatization, etc. in the middle stage (Zhang et al. 2020). It has been disclosed in previous studies that individuals' mental health directly affects their subjective well-being, which means that nurses' mental health also affects their subjective wellbeing and the COVID-19 epidemic significantly reduces their subjective well-being (Mukhtar 2020). 
Time management disposition (TMD) refers to a personality trait an individual displays in the way of using time in the dimensions of time, including time value, view of time monitoring and sense of time efficacy ( $\mathrm{Du}$ et al. 2012). Time value is the basis of an individual's time management, reflects the individual's stable attitude and concept about the function and value of time, and drives the individual to act towards a certain goal. At present, relevant researches focus on the relationship between time management and learning, social functions, physical and mental health, personality traits, etc. For example, Zhao et al. (2012) found that each dimension of time management disposition is significantly negatively correlated with job burnout, and significantly positively correlated with social adaptability. In other words, those with a low score of time management disposition would be more passive and more prone to the loss of many opportunities. As a consequence, negative emotions such as depression and anxiety are triggered, leading to poor social adaptation (Zhao 2012). Kaya et al. (2020) observed a significant negative correlation between time management ability and anxiety level in their analysis. Reasonable and effective use of time can significantly predict the mental health level (Kaya et al. 2020). It has been confirmed in subsequent related studies that time management skills can be improved through training. In the process of training college students to manage their spare time, some researchers found that their quality of life can be improved (Bi 2011). Meanwhile, time management, in the form of group counseling, can also promote individuals' learning satisfaction and self-efficacy. In addition, time management training can raise the mental health level among middle school students and alleviate their anxiety.

In conclusion, the COVID-19 epidemic has produced negative effects on nurses' mental health and subjective well-being. Therefore, it is significant to intervene in nurses' mental health in accordance with relevant theories. It has been proven in previous studies that time management intervention can elevate job satisfaction and boost one's mental health. However, there is no research that focuses on the intervention effect of time management training on the mental health of clinical nurses under the background of the epidemic. Meanwhile, the psychological intervention method of Balint Group has been verified to be effective in reducing negative emotional experience in doctor-patient interaction, and helping nurses better identify, understand and deal with negative emotions perceived during working ( $\mathrm{Li}$ et al. 2016). Therefore, the advantages of the two intervention methods were innovatively synthesized in this study to carry out comprehensive psychological intervention in nurses in the epidemic period and investigate the application effect of this model. The results of this study are expected to provide the management department with corresponding objective evidence to formulate the psychological care policy intended for nurses and offer reference for the effort made to improve medical practitioners' mental health during the epidemic.

\section{SUBJECTS AND METHODS}

\section{Participants}

Based on voluntary registration, 400 clinical nurses were randomly selected from three tertiary hospitals in Shanghai as the interviewees from September to December 2020. Inclusion criteria:

- those who have been working in clinical nursing for one year or longer;

- those who voluntarily participated in this study.

The participants were involved in the investigation of mental health. The second stage: After the explanation of the time training method and the Balint group psychological guidance and the explanation of the purpose and requirements of the study, the 66 nurses who participated in the intervention experiment voluntarily were divided into 35 members in the intervention group and 31 in the control group. All of them are female, aged 23-41 years old, with an average age of $34.12 \pm 4.87 ; 15$ of them have a bachelor degree and 51 have a college degree; there are 2 nurses in charge, 39 senior nurses and 25 nurses. The participants of this study all volunteered to participate in the intervention experiment, and were informed of the main contents, objectives and precautions of the intervention experiment before participating. All copies of the questionnaire were filled in anonymously.

\section{Methods}

From September to December 2020, the intervention was conducted once a week in a total of 8 weeks. Before and after the intervention, SCL-90, Campbell Index of Well-being and Work Stress Reaction Scale were used for measurement, to test the effect of the intervention experiment. Among them, the intervention group was given the time management training intervention in combination with Balint group. The control group did not receive the intervention designed in this study or any other professional psychological intervention.

\section{$1^{\text {st }}$ part}

The scheme of Balint group intervention: each activity was completed by 1 group leader and 8-10 members. The group leader should be a nursing worker who has obtained the psychological counselor qualification certificate, has participated in relevant training and passed the assessment of Balint group, and has hosted Balint group more than 10 times.

- The group leader took 2 minutes to introduce the purposes, methods and precautions of the group activity.

- One case was shared in each activity, with the topic limited to an event occurring during the fight against COVID-19. The case provider took about 10 minutes to describe the details of the event happening to $\mathrm{him} / \mathrm{her}$, tried to express the emotional fluctuation and personal feeling that impressed him/her most, and raised his/her own problems. After his/her repor- 
ting was finished, other members of the group asked more about the details they wanted to know and gave the case provider their answers.

- Group members freely expressed their views and feelings about the case within 30 minutes, and the group leader encouraged them to share and affirmed their statements.

- The case provider was given 8 minutes to abstract the key points based on the speech of each group member, present his/her understanding and feelings of the case, and express gratitude to other group members.

- The group leader used 10 minutes to summarize and evaluate the activity and show gratitude. The Balint group activity was conducted once to twice a week, about 60 minutes each time. To avoid the aggregation of individuals, each group activity was conducted in the form of online meeting.

\section{$2^{\text {nd }}$ part}

The scheme of time management intervention. This scheme was designed based on the combination of the findings of previous studies on mental health and the characteristics of nurses' psychological and physiological development. It focuses on the core links of time management disposition, such as its three dimensions. The training activity was conducted in the classroom, and the trainer should be a psychological graduate with the experience of hosting group intervention training. After each activity, members were asked to write down their feelings of participating in the training. Corresponding homework was assigned to evaluate the training effect and adjust the training content flexibly. The training consisted of 8 classes, one class a week, about 40 minutes each time. The contents of each class included: setting up the correct concept of time value, improving the awareness of cherishing time, discussing ideas and plans of life, exploring the methods to realize dreams, etc.

\section{Instruments}

\section{Symptom Checklist 90 (SCL-90)}

SCL-90 was used to evaluate the mental health of nurses. Revised and translated by Wang (2017), this scale has been widely used to measure the mental health of adults over 14 years old. The scale covers 9 dimensions (i.e. somatization, compulsion, interpersonal sen sitivity, depression, anxiety, hostility, terror, paranoia and psychosis) and one other factor. A total of 90 items are included in the scale and are rated with Likert 5level scoring (0-4). The score of each factor is the sum of the score in each item of $t$ each factor divided by the number of corresponding items. A higher score indicates worse mental health.

\section{Campbell Index of Well-being}

Campbell Index of Well-being was used to measure subjective well-being. The scale consists of two parts: Index of Overall Emotion (8 items) and Index of Life Satisfaction (1 item). Each item is rated with 7-level scoring. The scoring method is: (the average score of the first eight items $)+($ the average score of the last item $) \times 1.1=$ the total score of the scale. The total score is 2.1-14.7 and can be divided into three grades: 2.1-6 reflects low wellbeing; 6.1-10 implies moderate well-being; 10.1-14.7 corresponds to high well-being (Shi et al. 2016).

\section{Work Stress Reaction Scale}

This scale was compiled by Wang \& He (2018) on the basis of Work Stress Evaluation Scale created by House and Rizzo. It mainly includes 15 specific items in three dimensions, namely psychological reaction, physiological reaction and behavioral reaction. Each item is rated with 0-4 points, with 0 indicating no. A higher score suggests the appearance of more serious symptoms.

\section{Statistical Analysis}

Statistical Processing: SPSS19.0 software package was used for statistical analysis. The quantitative data was expressed with $X \pm s$. Paired sample t-test was used for the comparative analysis of the intervention effect on each group, and independent sample t-test was used for the comparison between the two groups, with $\mathrm{P}<0.05$ indicating that the difference is statistically significant.

\section{RESULTS}

\section{Status of Nurses' Mental Health}

From table 1, it can be seen that the nine factors of the mental health of the 400 nurses surveyed are significantly higher than the national norms, except for no significant difference in hostility and paranoia.

Table 1. General survey results of mental health

\begin{tabular}{lcccc}
\hline & Participants $(\mathrm{n}=400)$ & Norm $(\mathrm{n}=1388)$ & $\mathrm{t}$ & $\mathrm{p}$ \\
\hline Somatization & $1.58 \pm 0.67$ & $1.37 \pm 0.48$ & 11.33 & 0.00 \\
Compulsion & $1.78 \pm 0.43$ & $1.62 \pm 0.58$ & 9.89 & 0.00 \\
Interpersonal sensitivity & $1.71 \pm 0.44$ & $1.65 \pm 0.51$ & 3.29 & 0.01 \\
Depression & $1.67 \pm 0.41$ & $1.50 \pm 0.59$ & 5.35 & 0.00 \\
Anxiety & $1.59 \pm 0.44$ & $1.39 \pm 0.43$ & 8.48 & 0.00 \\
Hostility & $1.57 \pm 0.54$ & $1.46 \pm 0.55$ & 1.21 & 0.19 \\
Terror & $1.59 \pm 0.39$ & $1.23 \pm 0.41$ & 7.39 & 0.00 \\
Paranoia & $1.49 \pm 0.44$ & $1.43 \pm 0.57$ & 0.92 & 0.81 \\
Psychosis & $1.45 \pm 0.48$ & $1.29 \pm 0.42$ & 4.19 & 0.01 \\
\hline
\end{tabular}


Table 2. Comparative $t$ test of mental health scores between the intervention group and the control group before and after the intervention $(\mathrm{M} \pm \mathrm{SD})$

\begin{tabular}{|c|c|c|c|c|}
\hline & Intervention group $(n=35)$ & Control group $(n=31)$ & $\mathrm{t}$ & $\mathrm{p}$ \\
\hline \multicolumn{5}{|c|}{ Total average score in mental health } \\
\hline Before intervention & $1.10 \pm 0.09$ & $1.12 \pm 0.08$ & -0.81 & 0.42 \\
\hline After intervention & $0.50 \pm 0.07$ & $1.10 \pm 0.11$ & -25.74 & 0.00 \\
\hline $\mathrm{t}$ & 32.21 & 0.60 & & \\
\hline $\mathrm{p}$ & 0.00 & 0.77 & & \\
\hline \multicolumn{5}{|l|}{ Somatization } \\
\hline Before intervention & $0.96 \pm 0.26$ & $1.05 \pm 0.39$ & -1.51 & 0.13 \\
\hline After intervention & $0.47 \pm 0.37$ & $1.00 \pm 0.53$ & -11.31 & 0.00 \\
\hline $\mathrm{t}$ & 8.78 & 0.86 & & \\
\hline $\mathrm{p}$ & 0.00 & 0.56 & & \\
\hline \multicolumn{5}{|l|}{ Compulsion } \\
\hline Before intervention & $1.53 \pm 0.41$ & $1.54 \pm 0.37$ & -1.07 & 0.29 \\
\hline After intervention & $1.00 \pm 0.37$ & $2.99 \pm 0.48$ & -11.42 & 0.00 \\
\hline $\mathrm{t}$ & 11.36 & 1.54 & & \\
\hline $\mathrm{p}$ & 0.00 & 0.90 & & \\
\hline \multicolumn{5}{|l|}{ Interpersonal sensitivity } \\
\hline Before intervention & $0.94 \pm 0.35$ & $1.02 \pm 0.33$ & -1.37 & 0.17 \\
\hline After intervention & $0.53 \pm 0.14$ & $0.97 \pm 0.27$ & -8.25 & 0.00 \\
\hline $\mathrm{t}$ & 7.91 & 0.66 & & \\
\hline $\mathrm{p}$ & 0.00 & 0.12 & & \\
\hline \multicolumn{5}{|l|}{ Depression } \\
\hline Before intervention & $1.00 \pm 0.24$ & $1.06 \pm 0.27$ & -0.96 & 0.34 \\
\hline After intervention & $0.49 \pm 0.17$ & $0.95 \pm 0.25$ & -8.49 & 0.00 \\
\hline $\mathrm{t}$ & 12.06 & 1.89 & & \\
\hline $\mathrm{p}$ & 0.00 & 0.12 & & \\
\hline \multicolumn{5}{|l|}{ Anxiety } \\
\hline Before intervention & $1.43 \pm 0.43$ & $1.32 \pm 0.32$ & 1.14 & 0.42 \\
\hline After intervention & $0.57 \pm 0.37$ & $1.32 \pm 0.31$ & -11.30 & 0.00 \\
\hline $\mathrm{t}$ & 11.31 & -0.09 & & \\
\hline $\mathrm{p}$ & 0.00 & 0.12 & & \\
\hline \multicolumn{5}{|l|}{ Hostility } \\
\hline Before intervention & $1.19 \pm 0.33$ & $1.10 \pm 0.40$ & -0.95 & 0.42 \\
\hline After intervention & $0.50 \pm 0.37$ & $1.21 \pm 0.33$ & -10.49 & 0.00 \\
\hline $\mathrm{t}$ & 11.17 & 1.23 & & \\
\hline $\mathrm{p}$ & 0.00 & 0.12 & & \\
\hline \multicolumn{5}{|l|}{ Terror } \\
\hline Before intervention & $0.98 \pm 0.31$ & $0.95 \pm 0.23$ & -0.34 & 0.42 \\
\hline After intervention & $0.44 \pm 0.31$ & $1.00 \pm 0.18$ & -11.17 & 0.00 \\
\hline $\mathrm{t}$ & 10.19 & -1.23 & & \\
\hline $\mathrm{p}$ & 0.00 & 0.22 & & \\
\hline \multicolumn{5}{|l|}{ Paranoia } \\
\hline Before intervention & $1.01 \pm 0.29$ & $1.03 \pm 0.31$ & -0.26 & 0.42 \\
\hline After intervention & $0.48 \pm 0.19$ & $0.97 \pm 0.30$ & -8.07 & 0.00 \\
\hline $\mathrm{t}$ & 8.27 & -1.17 & & \\
\hline $\mathrm{p}$ & 0.00 & 0.25 & & \\
\hline \multicolumn{5}{|l|}{ Psychosis } \\
\hline Before intervention & $0.98 \pm 0.29$ & $0.99 \pm 0.29$ & -0.28 & 0.42 \\
\hline After intervention & $0.50 \pm 0.19$ & $108 \pm 0.25$ & -10.68 & 0.00 \\
\hline $\mathrm{t}$ & 8.33 & -1.39 & & \\
\hline $\mathrm{p}$ & 0.00 & 0.17 & & \\
\hline
\end{tabular}




\section{Comparison of SCL-90 scores before and after the intervention}

Table 2 shows that there are no significant differences between the intervention group and the control group in the total mental health score and the scores in the 9 dimensions before the intervention, indicating that the two groups have the same baseline levels. After the intervention, there is a significant decrease in the total score of the intervention group and significant decreases in the scores in the 9 dimensions, while there are no significant decreases in the control group.

\section{Comparison of subjective well-being scores before and after the intervention}

Table 3 shows that there are no significant differences between the intervention group and the control group in the total subjective well-being score and the scores in its dimensions before the intervention, indicating that the two groups have the same baseline levels. After the intervention, there is a significant increase in the total subjective well-being score of the intervention group and significant increases in the scores in its dimensions, while there are no significant increases in the control group.

Table 3. Comparative $t$ test of subjective well-being between the intervention group and the control group before and after the intervention $(\mathrm{M} \pm \mathrm{SD})$

\begin{tabular}{lcccc}
\hline & Intervention group $(\mathrm{n}=35)$ & Control group $(\mathrm{n}=31)$ & $\mathrm{t}$ & $\mathrm{p}$ \\
\hline Total subjective well-being & & & & \\
Before intervention & $3.52 \pm 0.38$ & $3.55 \pm 0.33$ & -0.28 & 0.78 \\
After intervention & $5.07 \pm 0.48$ & $3.62 \pm 0.38$ & 13.28 & 0.00 \\
$\mathrm{t}$ & -14.12 & -0.72 & & \\
$\mathrm{p}$ & 0.00 & 0.48 & & \\
Index of overall emotion & & & & \\
Before intervention & $3.53 \pm 0.42$ & $3.56 \pm 0.30$ & -0.32 & 0.74 \\
After intervention & $5.12 \pm 0.47$ & $3.64 \pm 0.41$ & 13.47 & 0.00 \\
$\mathrm{t}$ & -15.61 & -0.77 & & \\
$\mathrm{p}$ & 0.00 & 0.47 & & \\
Life satisfaction & & & & \\
Before intervention & $3.45 \pm 1.22$ & $3.45 \pm 1.09$ & 0.02 & 0.98 \\
After intervention & $4.63 \pm 1.51$ & $3.40 \pm 1.12$ & 3.45 & 0.00 \\
$\mathrm{t}$ & -3.19 & -0.11 & & \\
$\mathrm{p}$ & 0.00 & 0.92 & & \\
\hline
\end{tabular}

Table 4. Comparative $t$ test of work stress reaction between the intervention group and the control group before and after the intervention $(\mathrm{M} \pm \mathrm{SD})$

\begin{tabular}{|c|c|c|c|c|}
\hline & Intervention group $(\mathrm{n}=35)$ & Control group $(n=31)$ & $\mathrm{t}$ & $\mathrm{p}$ \\
\hline \multicolumn{5}{|c|}{ Total work stress reaction score } \\
\hline Before intervention & $2.23 \pm 0.24$ & $2.13 \pm 0.23$ & 1.67 & 0.09 \\
\hline After intervention & $1.04 \pm 0.15$ & $2.15 \pm 0.18$ & -22.42 & 0.00 \\
\hline $\mathrm{t}$ & 20.88 & -0.28 & & \\
\hline $\mathrm{p}$ & 0.00 & 0.77 & & \\
\hline \multicolumn{5}{|l|}{ Physiological reaction } \\
\hline Before intervention & $1.50 \pm 0.30$ & $1.37 \pm 0.39$ & 0.98 & 0.33 \\
\hline After intervention & $1.08 \pm 0.37$ & $1.54 \pm 0.53$ & -4.92 & 0.00 \\
\hline $\mathrm{t}$ & 3.72 & -2.15 & & \\
\hline $\mathrm{p}$ & 0.00 & 0.04 & & \\
\hline \multicolumn{5}{|l|}{ Psychological reaction } \\
\hline Before intervention & $3.10 \pm 0.38$ & $2.98 \pm 0.38$ & 1.32 & 0.19 \\
\hline After intervention & $1.00 \pm 0.37$ & $2.99 \pm 0.48$ & -23.15 & 0.00 \\
\hline t & 23.88 & -0.12 & & \\
\hline $\mathrm{p}$ & 0.00 & 0.90 & & \\
\hline \multicolumn{5}{|l|}{ Behavioral reaction } \\
\hline Before intervention & $2.09 \pm 0.35$ & $2.06 \pm 0.41$ & 0.36 & 0.42 \\
\hline After intervention & $1.06 \pm 0.37$ & $1.92 \pm 0.48$ & -10.48 & 0.00 \\
\hline $\mathrm{t}$ & 13.26 & 1.59 & & \\
\hline $\mathrm{p}$ & 0.00 & 0.12 & & \\
\hline
\end{tabular}




\section{Comparison of work stress reaction scores before and after the intervention}

Table 4 shows that there are no significant differences between the intervention group and the control group in the total work stress reaction score and the scores in the three dimensions before the intervention, indicating that the two groups have the same baseline levels. After the intervention, there is a significant decrease in the total work stress reaction score of the intervention group and significant decreases in the scores in the three dimensions, while there are no significant decreases in the control group, and there is a significant increase in the score of the control group in physiological reaction in the second test.

\section{DISCUSSION}

\section{Analysis of the general status of nurses' mental health}

According to the results of this study, the 400 nurses have significantly higher levels than the national norms in the nine factors of mental health (expect hostility and paranoia). To some extent, this suggests that attention should be paid to nurses who basically have a low mental health level. This result is consistent with the finding of Khattak et al. (2020) and Ning et al. (2020) that medical care practitioners suffer from a low mental health level, especially depression, anxiety, terror, etc. The root cause lies in the sudden outbreak of COVID19. On the one hand, the COVID-19 epidemic has dramatically increased the workload of nurses who are fighting on the front line. Nursing itself is a special occupation which has higher requirements than other ordinary work. The outbreak of COVID-19 has posed much higher work requirements on nurses. On the other hand, COVID-19 is a kind of infectious disease that has not been cured yet. It is featured with strong contagiousness and relatively high lethality rate. These characteristics of the disease directly aggravate nurses' worry about their work and thus affect their mental health (Sampaio et al. 2020).

\section{Intervention effect of time management training on nurses' mental health}

According to the results of this study, there is no significant difference between the intervention group and the control group in the mental health level before the intervention. After the 16-week intervention, there is a significant increase in the mental health level of the intervention group, and there are significant decreases in the nine factors, but there are no significant changes in the control group before and after the intervention. It can be seen that time management training in combination with Balint group can effectively improve the mental health level of nurses. This result is consistent with the findings of previous studies on the intervention in nurses' mental health solely with time management training and the intervention in nurses' mental health solely with Balint group (Khattak et al. 2020). From the results of previous studies and the earlier investigation in this study (Purba 2020), it can be known that even in the fourth quarter of 2020 when the severity of the epidemic decreased to some extent, the mental health level of nurses was still not optimistic, which can be inferred from obvious symptoms of depression, anxiety, hostility and somatization. There are the following profound reasons for the decrease of nurses' mental health: first, the excessive work makes it difficult for nurses to coordinate well between work and life, resulting in the work-family conflict (Maben et al. 2020). Secondly, the emotional loss incurred when coping with work and the epidemic is the more fundamental reason for the decline of nurses' mental health level (Han et al. 2021).

Time management training assists nurses to allocate working hours reasonably, and their effective utilization of working hours can be greatly improved by making the schedule and work list (Fan et al. 2015). Moreover, time management training also helps nurses develop the habit of setting goals and striving to achieve these goals, which is conducive to boosting their self-efficacy. With the skills obtained from time management training, nurses can better deal with work tasks, make full use of working hours, have more spare time to rest and accompany their family members, and therefore obtain gains both in work and family. The Balint group intervention model plays an important role in reducing nurses' emotional experience of rejuvenation. The Balint group intervention activity first provides nurses with a platform to disclose their confusion and frustration in daily work and life and release the negative emotions they experience in their work and life. Secondly, the Balint group intervention also gives nurses more opportunities to get social support. Unconditional listening among group members allows nurses to perceive support from others, especially from those who are working in the same industry. Finally, the Balint group intervention also equips nurses with more alternative coping styles to deal with negative emotions reasonably.

\section{Intervention effect of time management training on nurses' subjective well-being}

According to the results of this study, there is no significant difference between the intervention group and the control group in subjective well-being before the intervention. After the intervention, there are significant increases in the intervention group in subjective well-being, index of overall emotion and life satisfaction, but there are no significant differences in the control group before and after the intervention. It can be seen that time management training in combination with Balint group can effectively improve the subjective well-being of nurses. The result is consistent with the findings of previous studies on the intervention in subjective well-being solely with time management 
training and the intervention in subjective well-being solely with Balint group (Popa-Velea et al. 2021). According to the analysis above, the uncoordinated allocation of work and family life, the failure to effectively handle tasks and the perception of negative emotions in work and life are the main reasons for the decrease of nurses' mental health. With the decrease of mental health, nurses tend to experience more negative emotions such as depression and anxiety, thus suffering from lower subjective well-being.

The reason why time management training in combination with the Balint group intervention can improve the subjective well-being of nurses is that on the one hand time management training helps nurses make effective use of working hours to some extent, and prevents the extension of working hours from causing the failure to supplement the psychological resources needed for meeting the work requirements. This intervention approach also imperceptibly improves nurses' self-efficacy towards work, which is conducive to enhancing their sensitivity to positive emotions (Mesurado et al. 2018). As a consequence of time management training, the frequency of spending family life time on work is greatly reduced. According to the theory of social support, family is an irreplaceable source for individuals to obtain social support. Therefore, the increase of time used in family life contributes to family harmony and also enables nurses to obtain more social support from their family members, thus raising their subjective well-being. On the one hand, the Balint group activity gives nurses more opportunities to vent negative emotions, and also assists them to master more effective ways to cope with negative emotions. Hence, the Balint group activity can improve nurses' subjective well-being.

\section{Intervention effect of time management training on nurses' work stress reaction}

According to the results of this study, there is no significant difference between the intervention group and the control group in work stress reaction before the intervention. The nurses in both groups are at a higher level of work stress reaction, indicating that nurses perceive great work stress during the epidemic. After the 16-week intervention, there are significant decreases in the intervention group in work stress reaction, physiological reaction, psychological reaction and behavioral reaction, but there are no significant changes in the control group before and after the intervention. It can be seen that time management training in combination with Balint group can reduce the stress reaction of nurses to some extent. The result is consistent with the findings of previous studies on the intervention in nurses' work stress reaction solely with time management training (Jiang et al. 2019).

Excessive work stress usually leads to some physiological reactions such as fatigue, loss of appetite and poorer sleep quality, etc., reduces nurses' interest in interpersonal interaction, increases their sensitivity to depression and tension, and makes them more prone to behavioral reactions such as work errors and impatience (Labrague et al. 2018). As aforementioned, overly heavy work tasks directly affect nurses' perception of work stress, and may result in the extension of working hours, the reduction of appropriate rest and the decline of entertainment time. According to the theory of selfconsumption of self-control resources, sleep and rest are crucial ways for individuals to recover mental capacity and increase self-control resources (Inzlicht \& Friese 2019). In the process of dealing with the relationship with patients, nurses may encounter patients' misunderstanding, refusal to cooperate and other situations, therefore experiencing more negative emotions. It has been suggested in past studies that the sick population is the service target of nursing. Especially during the COVID-19 epidemic, hospitals seem to be synonymous with danger and infectious diseases. The panic of patients themselves and the fear of COVID-19 aggravate nurses' perception of t negative emotions. As indicated by the theory of self-consumption, individuals themselves consume a large part of self-control resources in dealing with negative emotions and are thus more likely to experience negative emotions (Dang 2018). The joint effort of emotional consumption and job requirements further aggravates nurses' work stress reaction. The scheme of time management designed in this study exactly fits the actual life of nurses, therefore having higher external ecological validity (Goldsby et al. 2020). Time management training allows nurses to learn to make reasonable use of working hours and ensure that they have enough rest time to buffer fatigue and supplement psychological resources (Elsayed et al. 2018). With the decrease in negative emotions after the Balint group intervention, nurses would perform well in coping with their psychological reactions caused by work stress.

\section{CONCLUSION}

A new comprehensive psychological intervention method, namely time management training in combination with Balint group, was conducted in this study to boost nurses' mental health. The results show that this comprehensive psychological intervention method can effectively improve nurses' mental health level and subjective well-being, and significantly reduce their work stress reaction. It can be inferred from the results that time management training in combination with Balint group enables nurses to better cope with nursing work during the epidemic and promote their overall health level. Nevertheless, there are still some shortcomings in this study: firstly, the control group was given no intervention, so that it is impossible to compare the effects of a single psychological intervention and the comprehensive intervention; secondly, the participants have the same psychological level, and no intervention was given to those with serious psychological problems alone. Future research can be further optimized in these aspects. 


\section{Acknowledgements: None.}

\section{Conflict of interest: None to declare.}

\section{References}

1. Al Maqbali M, Al Sinani M, Al-Lenjawi B: Prevalence of stress, depression, anxiety and sleep disturbance among nurses during the COVID-19 pandemic: A systematic review and meta-analysis. Journal of Psychosomatic Research 2021; 141: 110343

2. Bi KK: Research on the cultivation of College Students' time management ability. Comparative Study of Cultural Innovation 2018; 2(29): 22-23

3. Davidson JE, Proudfoot J, Lee K, Terterian G, Zisook S: A longitudinal analysis of nurse suicide in the United States (20052016) with recommendations for action. Worldviews on Evidence-Based Nursing 2020; 17(1): 6-15

4. Dang J: An updated meta-analysis of the ego depletion effect. Psychological Research 2018; 82(4): 645-651

5. Du L, Zhang $Q$, Xiao JJ: The relationship among undergraduate nursing students' time management disposition, self-efficacy and anxiety. Chinese Journal of Nursing 2012; 47(5): 430-433

6. ELsayed SS, Ibrahim MM, Diab GM, Mohamed SM: Relationship between Time Management Skills and Time Wasters among Nurse Managers at Menoufia University Hospital. Menoufia Nursing Journal 2018; 3(1): 77-84

7. Fan HX, Wang X: Influence of Time-Management Disposition Training on Female Postgraduates' Anxiety, Depression and Subjective Well-being. China Journal of Health Psychology 2015; 23(2): 285-285

8. Goldsby E, Goldsby M, Neck CB, Neck CP: Under Pressure: Time Management, Self-Leadership, and the Nurse Manager. Administrative Sciences 2020; 10(3): 38

9. Han Q, Zheng B, Agostini M, Bélanger JJ, Gützkow B, Kreienkamp J, et al.: Associations of risk perception of COVID19 with emotion and mental health during the pandemic. Journal of Affective Disorders 2021; 284: 247-255

10. Hofmeyer A, Taylor R: Strategies and resources for nurse leaders to use to lead with empathy and prudence so they understand and address sources of anxiety among nurses practising in the era of COVID-19. Journal of Clinical Nursing 2021; 30: 298-305

11. Inzlicht M, Friese M: The Past, Present, and Future of Ego Depletion. Social Psychology 2019; 50:370-378

12. Jiang $R$, Liang $R$, Yan XX: Effect of ABC time management on occupational stress and stress reaction of junior nurses. Today Nurse 2019; 26:23-25

13. Kaya H, Kaya N, Palloş AÖ, Küçük L: Assessing timemanagement skills in terms of age, gender, and anxiety levels: A study on nursing and midwifery students in Turkey. Nurse Education in Practice 2012; 12(5): 284-288

14. Khattak SR, Saeed I, Rehman SU, Fayaz M: Impact of Fear of COVID-19 Pandemic on the Mental Health of Nurses in Pakistan. Journal of Loss and Trauma 2020; 26(5): 421-435

15. Labrague LJ, McEnroe-Petitte DM, Leocadio MC, Van Bogaert $P$, Cummings $G G$ : Stress and ways of coping among nurse managers: An integrative review. Journal of Clinical Nursing 2018; 27(7-8): 1346-1359
16. Li Q, Zhao B, Jin YQ. Zhu XM, Wang XX: Intervention of Balint Group Training to the Negative Emotion of Nursing Staff. Nursing Journal of Chinese Peoples Liberation Army 2016; 33(16): 34-36

17. Maben J, Bridges J: Covid-19: Supporting nurses' psychological and mental health. Journal of Clinical Nursing 2020; 29(15-16): 2742-2750

18. Mesurado B, Vidal EM, Mestre AL: Negative emotions and behaviour: The role of regulatory emotional self-efficacy. Journal of Adolescence 2018; 64: 62-71

19. Mosolova E, Chung S, Sosin D, Mosolov S: Stress and Anxiety among Healthcare Workers Associated with COVID-19 Pandemic in Russia. Psychiatr Danub 2020;32:549-556

20. Mukhtar PS: Mental Wellbeing of Nursing Staff during the COVID-19 Outbreak: A Cultural Perspective. Journal of Emergency Nursing 2020; 46(4): 426-427

21. Ning $X, Y u F$, Huang $Q$, Li X, Luo Y, Huang $Q$, et al.: The mental health of neurological doctors and nurses in Hunan Province, China during the initial stages of the COVID-19 outbreak. BMC Psychiatry 2020; 20(1): 436

22. Pappa S, Ntella V, Giannakas T, Giannakoulis VG, Papoutsi E, Katsaounou P: Prevalence of depression, anxiety, and insomnia among healthcare workers during the COVID-19 pandemic: A systematic review and meta-analysis". Brain Behavior and Immunity 2020; 88: 901-907

23. Popa-Velea O, Mihăilescu AI, Diaconescu LV, Gheorghe IR, Ciobanu AM: Meaning in Life, Subjective Well-Being, Happiness and Coping at Physicians Attending Balint Groups: A CrossSectional Study. International Journal of Environmental Research and Public Health 2021; 18(7): 3455

24. Purba AK: How should the role of the nurse change in response to covid-19?. Nursing Times 2020; 116(6): 25-28

25. Salopek-Žiha D, Hlavati M, Gvozdanović Z, Gašić M, Placento $H$, Jakić H, Klapan D, Šimić H: Differences in Distress and Coping with the COVID-19 Stressor in Nurses and Physicians. Psychiatr Danub 2020; 32(2):287-293

26. Sampaio F, Sequeira C, Teixeira L: Nurses' mental health during the Covid-19 outbreak: A cross-sectional study. Journal of Occupational and Environmental Medicine 2020; 62:783-787

27. Shi J, Song GQ, Wang WL, Qi YZ, Xie LF: The inlfuence of nurse-nurse collaboration to the subjective well-being of nurses. Chinese Nursing Management 2016; 16(8): 1040-1043

28. Wang C, Pan R, Wan X, Tan Y, Xu L, McIntyre RS, et al.: A longitudinal study on the mental health of general population during the COVID-19 epidemic in China. Brain, Behavior, and Immunity 2020; 87: 40-48

29. Wang YL, He XM: Correlation analysis on career status and job stressors and psychological capital among "post-90s"nurses. Occupation and Health 2018; 34(4): 501-503

30. Wang ZH, Yu WL, Shen Z, Ye Y, Hu L, Yu GX, Sun LG: Reliability and validity of the symptom checklist 90 in Chinese professional females. Chinese Journal of Industrial Medicine 2017; 30(4): 247-250

31. Zhang Y, Wei LL, Li HT, Pan YS, Wang JY, Li QQ, et al.: The psychological change process of frontline nurses caring for patients with COVID-19 during its outbreak. Issues in Mental Health Nursing 2020; 41:525-530

32. Zhao WY, Jiang HF, Ji F: The relationship between time management disposition and coping style of College Students. China Journal of Health Psychology 2012; 20:589-591

Correspondence:

Liuyu Sun

School of Economics \& Management, Tongji University

Shangha, China

E-mail: SUN15021652598@126.com 